PROCEEDINGS OF THE

AMERICAN MATHEMATICAL SOCIETY

Volume 138, Number 8, August 2010, Pages 2939-2947

S 0002-9939(10)10291-3

Article electronically published on April 15, 2010

\title{
BOREL ORACLES. AN ANALYTICAL APPROACH TO CONSTANT-TIME ALGORITHMS
}

\author{
GÁBOR ELEK AND GÁBOR LIPPNER
}

(Communicated by Julia Knight)

\begin{abstract}
In 2008 Nguyen and Onak constructed the first constant-time algorithm for the approximation of the size of the maximum matching in bounded degree graphs. The Borel oracle machinery is a tool that can be used to convert some statements in Borel graph theory to theorems in the field of constant-time algorithms. In this paper we illustrate the power of this tool to prove the existence of the above mentioned constant-time approximation algorithm.
\end{abstract}

\section{INTRODUCTION}

1.1. Borel graphs. Borel graphs were introduced by Kechris, Solecki and Todorcevic in $[8$. Let $X$ be a standard Borel space, say the $[0,1]$ interval, with its Borel structure. Let $E \subset X^{2}$ be a Borel graph relation, that is, a Borel subset such that $(x, y) \in E$ implies $(y, x) \in E$ and $(x, x) \notin E$ for any $x \in X$. The $\mathcal{G}=(X, E)$ system is called a Borel graph. In this paper we are interested in Borel graphs in which all the degrees are bounded. Kechris, Solecki and Todorcevic proved that in such graphs there exists a maximal independent Borel subset, and using that they proved that if the degrees are bounded by $d$, then there exists a Borel coloring $c: X \rightarrow\{1,2, \ldots, d+1\}$ of the vertices. In order to appreciate this result let us note that the analogue of Vizing's theorem, the existence of a Borel edge-coloring $c: E \rightarrow\{1,2, \ldots, d+1\}$, is still unknown. Also, there exist some Borel trees with countable degrees such that they do not have countable Borel colorings (of course they have non-Borel colorings with only two colors).

A Borel matching in $\mathcal{G}(X, E)$ is a Borel set $M \subset E$ such that all of its components are single edges. For a matching $M$ we shall denote by $X_{M}$ the Borel subset of $X$ that consists of those points that are matched by $M$. An augmenting path for $M$ is a finite path $x_{0}, x_{1}, \ldots, x_{2 k+1}$ such that

- $\left(x_{2 j} x_{2 j+1}\right) \notin M$ and $x_{2 j+1} x_{2 j+2} \in M$ for all $0 \leq j \leq k$.

- The two endpoints $x_{0}, x_{2 k+1} \notin X_{M}$.

Received by the editors July 18, 2009 and, in revised form, November 14, 2009

2000 Mathematics Subject Classification. Primary 03E15; Secondary 68R10.

Key words and phrases. Constant time algorithms, graph limits, Borel graphs, invariant measures, maximum matching.

The first author's research was sponsored by OTKA Grant 67867 .

The second author's research was sponsored by OTKA Grant 69062 .

(C)2010 American Mathematical Society Reverts to public domain 28 years from publication 
We shall say that the "length" of an augmenting path $x_{0}, x_{1}, \ldots, x_{2 k+1}$ is $k$, and we shall often refer to it as a $k$-augmenting path. One can also define a (one ended) infinite augmenting path in the same way. If $M$ is a matching and $P$ is the edge-set of an augmenting path, then $M^{\prime}=M \triangle P$ is also a matching, in fact it is larger in the sense that $X_{M} \subsetneq X_{M^{\prime}}$. The matching $M^{\prime}$ will often be referred to as $M$ improved by $P$.

Our result is about the existence of Borel matchings without short augmenting paths.

Proposition 1.1. Let $T>0$. Then any bounded-degree Borel graph contains a Borel matching $M$ that has no $k$-augmenting paths for any $k \leq T$.

One might think that the previous proposition implies the existence of Borel matchings without any finite augmenting paths. Nevertheless, we have a counterexample.

Proposition 1.2. There exists a Borel graph $\mathcal{G}$ such that all the Borel matchings of $\mathcal{G}$ contain augmenting paths of finite length.

1.2. Constant-time approximation algorithms. In practice, the most efficient algorithms are linear algorithms, that is, those that take time proportional to their input. However, in some cases, even to access and store the total input in real time is impossible. These technological problems led to the development of constant-time algorithms that use only a small (random) fraction of the input and still efficiently analyze massive data-sets (see [11] for a recent survey). First, let us explain what we mean by constant-time algorithms for bounded-degree graphs.

Fix a constant $d>0$ and denote the set of all finite simple graphs with vertex degree bound $d$ by $G r a p h_{d}$. Our goal is to construct a randomized algorithm that calculates the size of the maximum matching of graph $G \in G r a p h_{d}$ with high precision and high probability in constant time (that is, the running time of our algorithms should be independent on the size of the graphs). In general, let $P:$ Graph $_{d} \rightarrow \mathbb{R}$ be a graph parameter (e.g. the ratio of the maximal matching, maximal independent set, and minimal vertex cover). A constant time approximation of the parameter means that for any fixed $\varepsilon>0$ we have a tester that takes a graph $G$ as an input. Then:

- It gives random uniform real label to the vertices from the interval $[0,1]$.

- Then the tester randomly picks $C(\varepsilon)$ vertices of the graph and explores the $C(\varepsilon)$-neighbourhood of the chosen vertices.

- Based on the sampling the tester gives an estimate $P_{E}(G)$ for the parameter $P(G)$ of the maximum matching in such a way that

$$
\operatorname{Prob}\left(\left|P(G)-P_{E}(G)\right|>\varepsilon\right)<\varepsilon .
$$

Nguyen and Onak constructed the first constant-time approximation algorithm for the ratio of the maximum matching, that is, $m(G)=\frac{|M(G)|}{|V(G)|}$, where $M(G)$ is a maximum matching. Note that such approximation algorithms do not exist for the ratio of the maximal independent set [5] or the minimal vertex cover [4].

How can we see that such testers exist for a certain graph parameter? The existence of constant-time algorithms can be translated to a topological statement using the notion of the Benjamini-Schramm graph convergence [3. Let us briefly recall the definition of graph convergence. A rooted $(r, d)$-ball is a finite, simple, connected graph $H$ such that 
- $\operatorname{deg}(y) \leq d$ if $y \in V(H)$.

- $H$ has a distinguished vertex $x$ (the root).

- $d_{G}(x, y) \leq r$ for any $y \in V(H)$.

For $r \geq 1$, we denote by $U^{r, d}$ the finite set of rooted isomorphism classes of rooted $(r, d)$-balls. Let $G(V, E)$ be a finite graph with vertex degree bound $d$. For $\alpha \in U^{r, d}, T(G, \alpha)$ denotes the set of vertices $x \in V(G)$ such that there exists a rooted isomorphism between $\alpha$ and the rooted $r$-ball $B_{r}(x)$ around $x$. Set $p_{G}(\alpha):=\frac{|T(G, \alpha)|}{|V(G)|}$. Thus we associated to $G$ a probability distribution on $U^{r, d}$ for any $r \geq 1$. Let $\left\{G_{n}\right\}_{n=1}^{\infty} \subset G r a p h_{d}$ be a sequence of finite simple graphs such that $\lim _{n \rightarrow \infty}\left|V\left(G_{n}\right)\right|=\infty$. Then $\left\{G_{n}\right\}_{n=1}^{\infty}$ is called convergent if for any $r \geq 1$ and $\alpha \in U^{r, d}, \lim _{n \rightarrow \infty} p_{G_{n}}(\alpha)$ exists. If $P$ is a graph parameter and $\lim _{n \rightarrow \infty} P\left(G_{n}\right)$ exists for any convergent graph sequence, then there exists a constant-time algorithm for the approximation of $P$ (Theorem 3, $[\underline{6}$ ).

Our main goal is to prove the following result.

Theorem 1.1. Let $\left\{G_{n}\right\}_{n=1}^{\infty} \subset G r a p h_{d}$ be a convergent graph sequence in the sense of Benjamini-Schramm. Then $\lim _{n \rightarrow \infty} m\left(G_{n}\right)$ exists.

By the previous remark, Theorem 1.1 implies that there exists a constant-time algorithm for approximating the maximum matching. Note that our result is highly non-constructive. In [10 an effective upper bound is given for the running time in terms of $d$ and $\varepsilon$. To prove the theorem we use Proposition 1.1 via the Borel oracle machinery. The machinery can be described informally in the following way. There is one single Borel graph with one given matching without short augmenting paths (The Borel Oracle). Sampling a finite graph can be viewed as "asking" the oracle about the optimal choice of picking a matching in this particular graph.

\section{The Proof of Propositions 1.1 and 1.2}

Let us start with a simple lemma which is a variation of the classical observation that a matching in a finite graph without augmenting paths is always of maximum size ([2]; see also Lemma 6 in [10]).

Lemma 2.1. Let $G$ be a finite graph. Let $M$ be a matching such that there are at most $q \cdot|G|$ vertices from which an augmenting path shorter than $T$ starts and let $M_{m}$ be a maximum size matching of $G$. Then

$$
\frac{|M|}{|G|} \leq \frac{\left|M_{m}\right|}{|G|} \leq \frac{|M|}{|G|} \cdot \frac{T+1}{T}+q
$$

Proof. Let $Q \subset G$ denote those vertices from which a short augmenting path starts. Consider the symmetric difference of the edge set of $M$ and $M_{m}$. This clearly consists of disjoint paths and cycles in which the edges coming from $M$ and $M_{m}$ are alternating. Hence cycles have to be of even length. In each cycle and in each path of even length there are just as many edges coming from $M$ as from $M_{m}$. On the other hand, if $x_{0}, x_{1}, \ldots, x_{2 k+1}$ is a path of odd length, then $x_{0} x_{1}$ must come from $M_{m}$ (and then $x_{2 k} x_{2 k+1}$ also). Otherwise it would be an augmenting path for $M_{m}$, which is clearly impossible. But then it must be an augmenting path for $M$, and so either $x_{0} \in Q$ or $k \geq T$ by our assumption. In the second case there are at least $T$ edges of $M$ in the path. Thus we have

$$
\left|M_{m}\right|-|M|=\text { number of odd paths in } M \triangle M_{m} \leq|Q|+\frac{|M|}{T} .
$$


Hence the lemma follows.

We start the proof of Proposition 1.1 by analysing the case of one single countable, connected graph. The construction for this case will be used in the case of Borel graphs too.

Lemma 2.2. Let $G(V, E)$ be a countable, connected graph with degree bound d, and let $T>0$. Then $G$ contains a matching $M$ that has no $k$-augmenting paths for any $k \leq T$.

Proof. Let us enumerate the vertices of $G$ such that each vertex is listed infinitely many times. Let $v_{1}, v_{2}, \ldots$ be this enumeration. We construct $M$ as a limit of a sequence of matchings $M_{0}, M_{1}, M_{2}, \ldots$ We start with the empty matching $M_{0}$. In the $i$-th step we take $M_{i-1}$. We look at the $4 T$-neighborhood $B_{4 T}\left(v_{i}\right)$ of $v_{i}$ in $G$. This is a finite subgraph of size at most $(d+1)^{4 T}$. We look for $k$-augmenting paths for $M_{i-1}$ of length at most $k \leq T$ in this subgraph, and we improve $M_{i-1}$ every time we find one. Since each improvement increases the number of edges of $M_{i-1}$ in $B_{4 T}\left(v_{i}\right)$, this process terminates in a finite number of steps. Let $M_{i}$ denote the improved matching.

Claim 2.1. The sequence $M_{i}$ stabilizes in the sense that for each edge $(x y) \in E$ there is an integer $N$ such that $(x y) \in M_{n}$ for all $n \geq N$ or $(x y) \notin M_{n}$ for all $n \geq N$. Hence we can define $M$ to be the (edge-wise) limit of $M_{i}$.

To see this it is enough to show that the "status" of a fixed edge can change only finitely many times during the process. Each change of status involves improving by an augmenting path of length at most $T$. The endpoints of such a path necessarily lie in $B_{2 T+1}(x)$. At each improvement, the number of matched vertices in this neighborhood strictly increases. Since the neighborhood is finite, there can be only finitely many improvements affecting the edge $(x y)$.

Claim 2.2. $M$ is a matching without any $k$-augmenting paths for any $k \leq T$.

Suppose there is a $k$-augmenting path $x_{0}, \ldots, x_{2 k+1}$ for $M$. By the previous claim we can choose $N$ so large that for any $n \geq N$ we have $\left.M\right|_{B_{4 T}\left(x_{0}\right)}=\left.M_{n}\right|_{B_{4 T}\left(x_{0}\right)}$. But there will also be an $n \geq N$ for which $v_{n}=x_{0}$. Then in the $n$-th step the path $x_{0}, \ldots, x_{2 k+1}$ has to be a $k$-augmenting path for $M_{n}$ in the $4 T$-neighborhood of $v_{n}$, so $\left.M_{n}\right|_{B_{4 T}\left(x_{0}\right)} \neq\left. M_{n+1}\right|_{B_{4 T}\left(x_{0}\right)}$, which is clearly a contradiction. A similar (but much simpler) argument shows that $M$ is in fact a matching.

Remark 2.3. Note that instead of improving one neighborhood in each step, it is perfectly fine if in each step we improve many (even infinitely many) disjoint $4 T$-neighborhoods.

Proof of Proposition 1.1. Let us color (in a Borel way) the vertices of our graph $\mathcal{G}$ with finitely many $(K)$ colors such that any two vertices of the same color have disjoint $4 T$-neighborhoods. This can be done by the result of Kechris, Solecki and Todorcevic. Indeed, let $\mathcal{G}^{\prime}$ be the Borel graph that we obtain if we connect two vertices in $\mathcal{G}$ when their distances are not greater than $4 T$ and consider a Borel coloring of $\mathcal{G}^{\prime}$ by finitely many colors.

Now we execute the algorithm described in the previous lemma in the following way: let $M_{0}$ be the empty matching. In step $n$ we take the matching $M_{n}$ constructed so far and take the $n$-th color class modulo $K$. We improve the matching $M_{n}$ in 
the $4 T$-neighborhood of each vertex of the color class parallel, just as in the lemma. Hence we obtain a sequence of matchings $M_{1}, M_{2}, \ldots$ Each $M_{n}$ will be Borel, since one step can be carried out in a Borel way: there are only finitely many different $4 T$-neighborhoods together with a matching. On each such neighborhood-withmatching we can fix a unique way of improving the matching, and hence each local improvement depends only on the starting state of the neighborhood of the vertex, which on the other hand depends on the vertex in a Borel way. There could be one more issue: the neighborhoods might have symmetries and then the choice of improvement might not be unique. However, using the colorings of the vertices we can order the augmented paths in the neighborhoods and always improve the first augmented paths to break the tie in case of a symmetry.

Now restricting this sequence to any single connected component of $G$ we get a sequence of matchings that we constructed in the lemma. Hence the sequence stabilizes and the limit $M$ will be a Borel matching without any $k$-augmenting paths for any $k \leq T$.

Now we prove Proposition 1.2. Recall that Laczkovich 9 ] constructed a 2-regular Borel graph $\mathcal{G}(X, E)$ on $X=[0,1]$ such that for any matching $M \subset E, \mu\left(X_{M}\right)<1$, where $\mu$ denotes the Lebesgue measure. Assume now that $M \subset E$ is a matching without finite augmenting paths, and let $X_{0}=X \backslash X_{M}$. If $x_{0} \in X_{0}$ and $x_{0}, x_{1}, x_{2}, \ldots$ is a path in $\mathcal{G}$, then $\left(x_{1} x_{2}\right)$ has to be in $M$ (since that graph is 2regular and $x_{0} x_{1}$ is not a 0 -augmenting path), $x_{3} x_{4}$ has to be in $M$ for a similar reason, and so on. It follows that $x_{1}, x_{2}, \ldots \in X_{M}$. Also if $x_{0} \neq y_{0} \in X_{0}$ and $y_{0}, y_{1}, y_{2}, \ldots$ is a path in $\mathcal{G}$, then $y_{1}, y_{2}, \ldots \in X_{M}$ and the $x_{i}$ 's are all different from the $y_{j}$ 's; otherwise there would again be a finite augmenting path. Therefore, from every $x \in X_{0}$ an infinite path in $X_{M}$ starts, and these paths are all disjoint. In fact, from each $x$ there are two such paths, starting with either edge incident to $x$. Let us choose the one which starts with the smaller vertex. Thus we have Borel maps $T^{i}: X_{0} \rightarrow[0,1]$ such that if $x_{0} \in X_{0}$, then $T^{i}\left(x_{0}\right)=x_{i}$. By our construction, $\mu\left(T^{i}\left(X_{0}\right)\right)=\mu\left(X_{0}\right)$ and $T^{i}\left(X_{0}\right) \cap T^{j}\left(X_{0}\right)=\emptyset$ if $i \neq j$. Hence $\mu\left(X_{0}\right)=0$, leading to a contradiction.

\section{INVARIANT MEASURES ON BOREL GRAPHS AS THE LIMITS OF FINITE GRAPHS}

In [3], Benjamini and Schramm constructed unimodular measures on the space of connected, countable graphs as the limit objects of convergent graph sequences. In this paper we consider invariant measures on Borel graphs as limit objects. The two notions are closely related (see Example 9.9, [1).

First of all, let us recall the notion of invariant measures on Borel graphs from 7 . Let $\mathcal{G}(X, E)$ be a Borel graph. A Borel involution $T: X \rightarrow X$ defines a matching of $\mathcal{G}$ if for any $x \in X, x \neq T(x): x$ is connected to $T$ in $\mathcal{G}$. A probability measure on $X$ is $\mathcal{G}$-invariant if it is preserved by all Borel involutions $T$ that define a matching in $\mathcal{G}$. Note that for the Borel graph in the proof of Proposition 1.2 the Lebesgue measure is an invariant probability measure.

Now let us recall the limit object construction from [6]. Let $B=\{0,1\}^{\mathbb{N}}$ be the Bernoulli space of 0-1-sequences with the standard product measure $\nu$. A (rooted) $B$-graph is a (rooted) graph $G$ and a function $\tau_{G}: V(G) \rightarrow B$. Two rooted $B$ graphs $G$ and $H$ are said to be isomorphic if there exists a rooted isomorphism $\psi: V(G) \rightarrow V(H)$ such that $\tau_{H}(\psi(x))=\tau_{G}(x)$ for every $x \in V(G)$. The set 
of isomorphism classes of all countable rooted $B$-graphs with degree bound $d$ is denoted by $\mathbf{B G r} \mathbf{r}_{d}$. In fact it will be more convenient to use the subset of this in which the labels are required to be different for every vertex in the graph. This subset shall be denoted $\mathbf{B}_{d}$.

Let $U^{r}=U^{r, d}$ denote the set of isomorphism classes of rooted $r$-balls with degree bound $d$ and vertices labeled with $\{0,1\}^{r}$. For a $B$-graph $B G$ and a vertex $x \in$ $V(B G)$ by $B_{r}(x) \in U^{r}$, we shall denote the rooted $r$-ball around $x$ with the labels truncated to the first $r$ digits. For any $\alpha \in U^{r}$ and a finite $B$-graph $B G$, we define the set $T(B G, \alpha) \stackrel{\text { def }}{=}\left\{x \in V(G): B_{r}(x) \cong{ }_{B} \alpha\right\}$ and define $p_{B G}(\alpha) \stackrel{\text { def }}{=} \frac{|T(B G, \alpha)|}{|V(G)|}$. For $\alpha \in U^{r}$ let us define

$$
T\left(\mathbf{B G r}_{d}, \alpha\right):=\left\{X \in \mathbf{B G r}_{d}: \text { where for the root } x \text { of } X: B_{r}(x) \cong \alpha\right\} .
$$

There is a natural metric on $\mathbf{B G} \mathbf{r}_{d}$. If $X, Y \in \mathbf{B G} \mathbf{r}_{d}$, then

$$
d_{b}(X, Y)=2^{-r},
$$

where $r$ is the maximal number such that $B^{r}(x) \cong B^{r}(y), x$ is the root of $X$, and $y$ is the root of $Y$. The subsets $T\left(\mathbf{B G r}_{d}, \alpha\right): \alpha \in U^{r}, r \in \mathbb{N}$, are clopen sets and generate the Borel-structure of $\mathbf{B G} \mathbf{r}_{d}$.

Let $\left\{B G_{n}\right\}_{n=1}^{\infty}$ be a sequence of $B$-graphs. We say that $\left\{B G_{n}\right\}_{n=1}^{\infty}$ converges if for any $\alpha \in U^{r}, \lim _{n \rightarrow \infty} p_{B G_{n}}(\alpha)=\mu\left(T\left(\mathbf{B G r}_{d}, \alpha\right)\right)$ exists. In this case $\mu$ naturally extends to a Borel-measure on $\mathbf{B G} \mathbf{r}_{d}$. We call $\mu$ the limit measure of $\left\{B G_{n}\right\}_{n=1}^{\infty}$.

$\mathbf{B G} \mathbf{r}_{d}$ can be given a Borel graphing structure $\mathcal{G}$ in a natural way: two rooted $B$-graphs $B G$ and $B H$ with roots $x \in B G, y \in B H$ are adjacent if there is a vertex $z \in B G$ adjacent to $x$ such that $B G$ with root $z$ is isomorphic to $B H$ with root $y$. Obviously this is a Borel graph with degree bound $d$. The following proposition is the straightforward consequence of Proposition 2.2 and Corollary 3.1 of [6] (see also Example 9.9 of [1]).

Proposition 3.1. Let $\left\{G_{n}\right\}_{n=1}^{\infty}$ be a convergent graph sequence. Let $\left\{B G_{n}\right\}_{n=1}^{\infty}$ be a uniformly random $B$-labelling of the vertices of $G_{n}$. Then $\left\{B G_{n}\right\}_{n=1}^{\infty}$ almost surely converges to a measure $\mu$ which is concentrated entirely on $\boldsymbol{B}_{d}$, and restricted to this subset it is $\mathcal{G}$-invariant.

\section{Proof of Theorem 1.1}

In this section $\mathcal{G}$ denotes the natural Borel graph on $\mathbf{B}_{d}$. Let $\left\{G_{n}\right\}_{n=1}^{\infty}$ be a convergent graph sequence. Then by Proposition 3.1 there is a $B$-labeling of the vertices so that the resulting $B$-graph sequence $\left\{B G_{n}\right\}_{n=1}^{\infty}$ converges to a $\mathcal{G}$ invariant measure $\mu$ on $\mathbf{B}_{d}$.

Let us fix an $\varepsilon>0$ and choose an integer $T>1 / \varepsilon$. By Proposition 1.1 there exists a Borel matching $M$ of the graphing $\mathcal{G}$ that has no $t$-augmenting paths for any $t \leq T$.

This matching $M$ is our Borel oracle. Using $M$ we will be able to construct matchings $M_{n}$ of $G_{n}$ that almost have the same property. Any point $x \in \mathbf{B}_{d}$ represents a labeled, rooted graph. Let $l(x)$ denote the label of the root of this graph. If the neighbors of $x$ are $y_{1}, y_{2}, \ldots, y_{s}$, then $l\left(y_{1}\right), \ldots, l\left(y_{s}\right)$ are exactly the labels of the neighbors of the root of $x$. Since all the labels of the graph are different, 
we can order $y_{1}, \ldots, y_{s}$ according to their $l$-values. If $l\left(y_{1}\right)<l\left(y_{2}\right)<\cdots<l\left(y_{s}\right)$, then $y_{i}$ will be called the $i$-th neighbor of $x$. Let $X_{0}=\mathbf{B}_{d} \backslash\left(\mathbf{B}_{d}\right)_{M}$ and let

$$
\begin{aligned}
X_{i j}=\left\{x \in\left(\mathbf{B}_{d}\right)_{M}: x \text { is connected to its } i\right. \text {-th neighbor in the } \\
\text { matching } M \text {, and } x \text { is the } j \text {-th neighbor of its matched vertex }\} .
\end{aligned}
$$

Therefore if $x y \in M$ and $x \in X_{i j}$, then $y \in X_{j i}$. This way we get a Borel partition of $\mathbf{B}_{d}$ into $d^{2}+1$ sets. It is easy to see that this partition can be approximated by another one (denoted by $X_{0}^{\prime} \cup \bigcup X_{i j}^{\prime}$ ) in which each part is a finite union of clopen sets, and that the total symmetric difference $H=\bigcup_{1 \leq i, j \leq d}\left(X_{i j} \triangle X_{i j}^{\prime}\right) \cup\left(X_{0} \triangle X_{0}^{\prime}\right)$ is so small that $\mu(H) \leq \frac{\varepsilon}{(d+1)^{2 T+1}}$. Since the approximating sets are finite unions of clopen sets, this means that there is an $r$ such that for any $x$ the neighborhood $B_{r}(x)$ determines which $X_{i j}^{\prime}$ contains $x$. Since we are in $\mathbf{B}_{d}$, by choosing $r$ large enough we may assume that we only use such neighborhoods in which all the $r$-digit labels are different. (More precisely, those neighborhood types for which this does not hold can be put into $H$.)

Now let us look at a vertex $v \in B G_{n}$ and look at its $B_{r}(v)$ neighborhood. Let us find the unique $X_{i j}^{\prime}$ (or $X_{0}^{\prime}$ ) that contains the points having this neighborhood. If it is in $X_{i j}^{\prime}$, then we look at its $i$-th neighbor $w$ and look at $B_{r}(w)$. If $B_{r}(w)$ is contained in $X_{j i}^{\prime}$ and $v$ is indeed the $j$-th neighbor of $w$, then we match $v$ to $w$. Otherwise we don't match $v$ at all. This way we obtain a matching $M_{n}$ of $B G_{n}$ (and of $G_{n}$ of course). Indeed, since $\left\{X_{i j}^{\prime}\right\} \cup X_{0}^{\prime}$ is a partition the degrees of any vertex in the subgraph, $M_{n}$ can be either 0 or 1 .

We claim that there cannot be too many vertices that are endpoints of a $t$ augmenting path in the matching $M_{n}$ if $t \leq T$. Let $v_{0}, v_{1}, \ldots, v_{2 t+1}$ be a $t$ augmenting path in $B G_{n}$. Let $\alpha \in U^{r+2 t+1}$ denote the isomorphism type of $B_{r+2 t+1}\left(v_{0}\right)$.

Claim 4.1. If $x_{0} \in \mathbf{B}_{d}$ and $B_{r+2 t+1}\left(x_{0}\right) \equiv \alpha$, then $x_{0} \in H^{2 t+1}$, where $H^{2 t+1}$ denotes the set of points that are at most $2 t+1$ steps from $H$ in the Borel graph $\mathcal{G}$.

Indeed, lift the path $v_{0}, v_{1}, \ldots, v_{2 t+1}$ in $B G_{n}$ to a path $x_{0}, x_{1}, \ldots, x_{2 t+1}$ in $\mathbf{B}_{d}$ by always finding the appropriate neighbor of the vertices according to the ordering respectively. If no point of the lifted path falls into $H$, then by the construction the matching $M$ looks precisely the same along this path as $M_{n}$ looked along the $v$-path in $B G_{n}$. However, this is not possible, as $M$ does not contain a $t$-augmenting path. Hence one of the $x_{i}$ 's has to be in $H$, or in other words $x_{0} \in H^{2 t+1}$, proving the claim.

We will need the following lemma.

Lemma 4.1. $\mu\left(H^{2 T+1}\right) \leq(d+1)^{2 T+1} \cdot \mu(H)$.

Proof. It is enough to prove that $\mu\left(H^{1}\right) \leq(d+1) \mu(H)$. First, let us consider a Borel coloring of the edges by the natural numbers (see [7]). The set $H_{n} \subset H$ is defined as follows. A point $p \in H$ is in $H_{n}$ if and only if there is a point $q \notin H$ such that $(p, q) \in E$ and the color of $(p, q)$ is $n$. The set of all such points $q$ will be denoted by $K_{n}$. By the invariance of $\mu, \mu\left(H_{n}\right)=\mu\left(K_{n}\right)$. For a $k$-tuple $Q=\left\{i_{1}, i_{2}, \ldots, i_{k}\right\}$, where $0 \leq k \leq d$ and $i_{1}<i_{2}<\cdots<i_{k}$, let $H_{Q}$ be defined as the points in $H$ that are exactly in the sets $H_{i_{1}}, H_{i_{2}}, \ldots, H_{i_{k}}$. Clearly, $\bigcup_{Q} H_{Q}=H$. 
Now let $K_{Q}^{i_{j}}$ be the set of points outside $H$ that are connected to a point in $H_{Q}$ by an edge colored by $i_{j}$. By the invariance of $\mu, \mu\left(\bigcup_{i_{j} \in Q} K_{Q}^{i_{j}}\right) \leq d \mu\left(H_{Q}\right)$. Since $H^{1}=H \cup\left(\bigcup_{Q} \bigcup_{i_{j} \in Q} K_{Q}^{i_{j}}\right)$, our lemma follows.

We call $v \in B G_{n}$ a bad point if there is a $t$-augmenting path (for any $t \leq T$ ) starting from $v$, and we denote by $Q_{n} \subset B G_{n}$ the set of bad points and let $q_{n}=$ $\left|Q_{n}\right| /\left|V\left(G_{n}\right)\right|$. Let $A \subset U^{r+2 T+1}$ denote the set of neighborhood types of this size of all the bad points in all the $B G_{n}$ 's. Then by the previous claim $\bigcup_{\alpha \in A} T\left(\mathbf{B}_{d}, \alpha\right) \subset$ $H^{2 T+1}$. Hence we have

$$
\begin{aligned}
\limsup q_{n} \leq \limsup & \sum_{\alpha \in A} p_{B G_{n}}(\alpha)=\sum_{\alpha \in A} \lim p_{B G_{n}}(\alpha) \\
& =\mu\left(\bigcup_{\alpha \in A} T\left(\mathbf{B}_{d}, \alpha\right)\right) \leq \mu\left(H^{2 T+1}\right) \leq(d+1)^{2 T+1} \cdot \mu(H) \leq \varepsilon .
\end{aligned}
$$

Let us introduce the notation $s_{n}:=\left|M_{n}\right| /\left|V\left(G_{n}\right)\right|$ and $m_{n}:=m\left(G_{n}\right)$.

Claim 4.2. $\lim _{n \rightarrow \infty} s_{n}=S$ exists.

Indeed, by definition, being a vertex in the matching depends only on the $r+1$ neighborhood type of the given vertex.

By Lemma 2.1 we have $s_{n} \leq m_{n} \leq s_{n} \cdot \frac{T+1}{T}+q_{n}$. Hence

$S=\liminf s_{n} \leq \liminf m_{n} \leq \limsup m_{n}$

$$
\leq \frac{T+1}{T} \limsup s_{n}+\lim \sup q_{n} \leq \frac{T+1}{T} S+\varepsilon,
$$

and thus

$$
\lim \sup m_{n}-\liminf m_{n} \leq \frac{1}{T} S+\epsilon \leq 3 \varepsilon .
$$

Since $\varepsilon$ can be arbitrarily small, it follows that the sequence $\left\{m\left(G_{n}\right)\right\}_{n=1}^{\infty}$ is convergent.

\section{ACKNOWLEDGEMENT}

We would like to thank Michael Krivelevich for calling our attention to [10.

\section{REFERENCES}

1. D. Aldous and R. Lyons, Processes on Unimodular Random Networks. Electron. J. Probab. 12 (2007), no. 54, 1454-1508. MR2354165 (2008m:60012)

2. A.S. Asratian, T.M.J. Denley And R. HÄGGkVist, Bipartite graphs and their applications. Cambridge Tracts in Mathematics, Cambridge University Press (1998). MR.1639013 (99e:05121)

3. I. Benjamini And O. Schramm, Recurrence of distributional limits of finite planar graphs. Electron. J. Probab. 6 no. 23 (2001) 13 pp. (electronic). MR 1873300(2002m:82025)

4. A. Bogdanov, K. Obata and L. Trevisan, A Lower Bound for Testing 3-Colorability in Bounded-Degree Graphs, 43rd Annual IEEE Symposium on Foundations of Computer Science (2002) 93-102.

5. B. BollobÁs, The independence ratio of regular graphs. Proc. Amer. Math. Soc. 83 no. 2 (1981) 433-436. MR624948 (82m:05079)

6. G. Elek, Parameter testing in bounded degree graphs of subexponential growth (to appear). http://arxiv.org/abs/0711.2800

7. A. Kechris And B. D. Miller, Topics in orbit equivalence theory. Lecture Notes in Mathematics, 1852. Springer-Verlag, Berlin, 2004. MR 2095154 (2005f:37010) 
8. A. Kechris, S. Solecki And S. Todorcevic, Borel chromatic numbers, Advances in Mathematics 141 no. 1 (1999) 1-44. MR.1667145 (2000e:03132)

9. M. Laczkovich, Closed sets without measurable matchings, Proc. of the AMS 103 no. 3 (1988) 894-896. MR.947676 (89f:28018)

10. H. N. NGUYEN AND K. ONAK, Constant-time approximation algorithms via local improvements, 49th Annual IEEE Symposium on Foundations of Computer Science (2008) 327-336.

11. R. Rubinfeld, Sublinear time algorithms, International Congress of Mathematicians. Vol. III, Eur. Math. Soc., Zürich (2006) 1095-1110. MR2275720 (2007m:68124)

Alfred Renyi Institute of the Hungarian Academy of Sciences, P.O. Box 127, H-1364, Budapest, Hungary

E-mail address: elek@renyi.hu

Department of Computer Science, Eötvös University, PÁzmány Péter sétány 1/C, H-117, Budapest, Hungary

E-mail address: lipi@cs.elte.hu 